\title{
CpG methylation controls reactivation of HIV from latency
}

\author{
Jana Blazkova ${ }^{1,2,3}$, Katerina Trejbalova ${ }^{1,2}$, Francoise Gondois-Rey ${ }^{1}$, Halfon Philippe ${ }^{4}$, Philibert Patrick ${ }^{5}$, \\ Eric Verdin ${ }^{6}$, Daniel Olive ${ }^{1}$, Carine van Lint ${ }^{3}$, Jiri Hejnar ${ }^{2}$, Ivan Hirsch ${ }^{1 *}$ \\ From $16^{\text {th }}$ International Symposium on HIV and Emerging Infectious Diseases \\ Marseille, France. 24-26 March 2010
}

\section{Background}

DNA methylation of retroviral promoter and enhancer localized in the provirus 5 ' long terminal repeat (LTR) is considered to be a mechanism of transcriptional suppression that allows retroviruses to evade host immune responses and antiretroviral drugs. However, the role of DNA methylation in the control of HIV-1 latency has never been unambiguously demonstrated, in contrast to the apparent importance of transcriptional interference and chromatin structure, and has never been studied in HIV-1-infected patients.

\section{Methods}

We analyzed the relation of latent and reactivated HIV1 promoters in a model of Jurkat cell lines and in memory $\mathrm{CD}^{+} \mathrm{T}$ cells of long-term aviremic patients by means of bisulfit sequencing and chromatin immunoprecipitation in cell-sorted populations. To assess the resistance of latent HIV-1 to reactivation we exposed the cells to TNF- $\alpha$, protein kinase $C$ agonists, inhibitors of HDAC, and inhibitors of DNA methyltransferases.

\section{Results}

We show in an in vitro model of reactivable latency and in a latent reservoir of HIV-1-infected patients that CpG methylation of the HIV-1 5' LTR is an additional epigenetic restriction mechanism, which controls resistance of latent HIV-1 to reactivation signals and thus determines the stability of the HIV-1 latency. CpG methylation acts as a late event during establishment of HIV-1 latency and is not required for the initial provirus silencing. Indeed, the latent reservoir of some aviremic patients contained high proportions of the non-methylated 5' LTR. In the

\footnotetext{
* Correspondence: ivan.hirsch@inserm.fr

${ }^{1}$ INSERM, UMR891, Centre de Recherche en Cancérologie de Marseille and
} Institut Paoli-Calmettes, and Université Méditerranée, Marseille, France latent reservoir of HIV-1-infected individuals without detectable plasma viremia, we found HIV-1 promoters and enhancers to be hypermethylated and resistant to reactivation, as opposed to the hypomethylated 5' LTR in viremic patients. However, even dense methylation of the HIV-1 5'LTR did not confer complete resistance to reactivation of latent HIV-1 with some histone deacetylase inhibitors, protein kinase $C$ agonists, TNF- $\alpha$, and their combinations with 5-aza-2deoxycytidine: The densely methylated HIV-1 promoter was most efficiently reactivated in virtual absence of $\mathrm{T}$ cell activation by suberoylanilide hydroxamic acid.

\section{Discussion}

The latency controlled solely by transcriptional interference and by chromatin-dependent mechanisms in the absence of significant promoter DNA methylation tends to be leaky and easily reactivable. Tight but incomplete control of HIV-1 latency by $\mathrm{CpG}$ methylation might have important implications for strategies aimed at eradicating HIV-1 infection.

\footnotetext{
Author details

${ }^{1}$ INSERM, UMR891, Centre de Recherche en Cancérologie de Marseille and Institut Paoli-Calmettes, and Université Méditerranée, Marseille, France. ${ }^{2}$ Institute of Molecular Genetics, Academy of Sciences of the Czech Republic, Prague, Czech Republic. ${ }^{3}$ Laboratory of Molecular Virology, Institute for Molecular Biology and Medicine (IBMM) University of Brussels (ULB), Gosselies, Belgium. ${ }^{4}$ Department of Virology, Alphabio Laboratory, Marseille, France. ${ }^{5}$ Department of Infectious Diseases, Hôpital Ambroise Paré, Marseille, France. ${ }^{6}$ Gladstone Institute of Virology and Immunology, San Francisco, USA.

Published: 11 May 2010

doi:10.1186/1742-4690-7-S1-08

Cite this article as: Blazkova et al.: CpG methylation controls reactivation of HIV from latency. Retrovirology 2010 7(Suppl 1):08.
} 